\title{
On the Existence and uniqueness for solution of system Fractional Differential Equations
}

\author{
Maha Abd Al-Wahab \\ Department of Applied Science University of Technology Baghdad-Iraq
}

Abstract: In this paper we extend theorem of existence and uniqueness of fractional differential equations to $n$ system of fractional differential equations.

\section{Introduction}

Fractional differential equation is a generalization of ordinary differential equations and integrations to arbitrary non integer order. For the past three centuries this subject has been dealt with by mathematicians and in the last years this essential to under stand the solution of many application in various fields of sciences like physics and engineering [1,2], chemistry and computer hard disc by control by [3,4], also nuclear energy science by [5,6] and dynamic systems [7]. This study is deals with existence and uniqueness of solutions for $\mathrm{n}$ system of fractional differential order equation of the form $y^{(\alpha)}(x)=\lambda f(y(x)) \quad x \in(a, \infty)$ with $y^{(\alpha-1)}(x)=\mu, \mu$ is some constant and $|\mathrm{y}(\mathrm{x})|<\exp \left(\alpha \mathrm{c}^{-1}|\mathrm{x}|\right) \cdot$ constant, choosing $\lambda$ such that $|\lambda|<e^{\alpha}\left(\left(\frac{c}{\alpha}\right)^{\alpha}\right)^{-1}$ and $\mathrm{f}(\mathrm{y}(\mathrm{x}))$ be a continuous function on $[\mathrm{a}, \infty), 0<\alpha \leq 1, \gamma$ be positive constant, $\mathrm{g} \in \mathrm{c}[\mathrm{a}, \infty)$ such that $|\mathrm{g}(\mathrm{x})| \leq|\mathrm{x}|+\mathrm{c}, \mathrm{x} \in[\mathrm{a}, \infty)$.

\section{Preliminaries}

In this section, we introduce notation, definitions and preliminary facts which are used through out this paper.

Definition (2-1) ([8]): Let $A=\left\{F: F:[a, \infty) \times R^{n} \rightarrow R\right.$ be continuous function $\}$

Such that $F(x, y)=\left(F_{1}(x, y), F_{2}(x, y), \ldots, F_{n}(x, y)\right)^{T}$, where $y=\left(y_{1}, y_{2}, \ldots, y_{n}\right)$ and $T$ is the transpose of $\left(F_{1}, F_{2}, \ldots, F_{n}\right)$. Let the norm $\|\cdot\|$ on A be defined by $\|F\|=\operatorname{Sup}_{x \in[a, \infty)}\left\{e^{-\gamma|y(x)|} \mid F(y(x) \mid\}\right.$, where

$|F(y(x))|=\sum_{i=1}^{n}\left(F_{i}^{2}(y(x))\right)^{\frac{1}{2}}$, provided that this norm exists for some $\gamma>0$.

Definition (2.2) ([8]):

a- $\quad$ Let $f$ be a lebesgue- measurable function define $a \cdot e$ on $[a, b]$. if $\alpha>0$ then we define

$I_{a}^{b} f=\frac{1}{\Gamma(\alpha)} \int_{a}^{b} f(t)(b-t)^{\alpha-1} d t$ provided the integral (Lebesgue) exists.

b- $\quad$ If $\alpha \in \mathrm{R}, \mathrm{f}$ is define $\mathrm{a} \cdot \mathrm{e}$ on $[\mathrm{a}, \mathrm{b}]$, we define

$$
\frac{d^{\alpha} f}{d x^{\alpha}}=f^{(\alpha)}(x)=\coprod_{a}^{x}{ }^{-\alpha} f \text { for all } \mathrm{x} \in[\mathrm{a}, \mathrm{b}] \text { provided that } I_{a}^{x} f \text { exists. }
$$

Lemma (2.1)([9]): Let $0<\alpha \leq 1$ and $f$, $g$ be a continuous function on $(a, \infty)$, where $a \in R$ and such that $\operatorname{Sup}\{|\mathrm{f}(\mathrm{g}(\mathrm{x}))|: \mathrm{x} \in(\mathrm{a}, \infty)\}=\mathrm{M}<\infty$. Define

$f_{\alpha}(x)=\frac{\mu(x-a)^{\alpha-1}}{\Gamma(\alpha)}+\frac{1}{\Gamma(\alpha)} \int_{a}^{x}(x-t)^{\alpha-1} f(g(t)) d t \quad$ for all $\mathrm{x}>\mathrm{a}$ and $\mu$ is some constant. Then $\mathrm{f}_{\alpha} \in$ $\mathrm{c}(\mathrm{a}, \infty)$. 
Lemma (2.2) ([8]): Suppose that $G$ is a banach space and let $T \in L(G)$ such that $\|T\|<1$. then $I-T$ is regular and $(I-T)^{-1}=I+\sum_{n=1}^{\infty} T^{n}$, where the series $\sum_{n} T^{n}$ converge in $\mathrm{L}(\mathrm{G})$.

Lemma (2.3)([9]): Let us define $F_{\alpha}(x)=(x-a)^{1-\alpha} f_{\alpha}(x)$ on $(a, \infty)$, where $f_{\alpha}$ defined in Lemma $(2.1)$ and $0<\alpha \leq 1$. then $\mathrm{F}_{\alpha} \in \mathrm{c}[\mathrm{a}, \infty)$.

Lemma (2.4) ([8]): Let $\alpha, \gamma \in \mathrm{R}, \gamma>-1$. If $\mathrm{x}>\mathrm{a}$ then ${\underset{a}{x}}^{\alpha} \frac{(t-a)^{\gamma}}{\Gamma(\gamma+1)}=\left\{\begin{array}{cc}\frac{(x-a)^{\alpha+\gamma}}{\Gamma(\alpha+\gamma+1)}, & \alpha+\gamma \neq \text { negative integer } \\ 0 \quad, \quad \alpha+\gamma=\text { negative integer }\end{array}\right\}$

Lemma (2.5) ([9]): If $0<\alpha \leq 1$ and $f(x)$ is continuous on (a,b], $|f(x)| \leq M$ for all $x \in(a, b]$ (where $M \in R^{+}, M>0$ ). Then $I_{a}^{x}{ }_{a}^{-\alpha} I^{\alpha} f=f(x)$ for all $\mathrm{x} \in(\mathrm{a}, \mathrm{b}]$.

\section{Main Results}

In this section we prove the existence and uniqueness solution of a system of fractional differential equations.

Theorem (3): Let $0<\alpha \leq 1$ and $\gamma$ be a positive constant. Let $g(x)=\left(g_{1}(x), g_{2}(x), \ldots, g_{n}(x)\right)^{T}, x \in[a, \infty)$, where $g_{i}$ are continuous on $[\mathrm{a}, \infty), \mathrm{i}=1,2, \ldots, \mathrm{n}$ and $|\mathrm{g}(\mathrm{x})|=\left(\sum_{i=1}^{n} g_{i}^{2}\right)^{\frac{1}{2}}$ and $|\mathrm{g}(\mathrm{x})| \leq \mathrm{x}+\mathrm{c}$

where $\mathrm{c}$ is a positive constant. Let $\mathrm{f}_{\mathrm{i}}=\left(\mathrm{f}_{1}, \mathrm{f}_{2}, \ldots, \mathrm{f}_{\mathrm{n}}\right)^{\mathrm{T}}$ such that $\mathrm{f}_{\mathrm{i}} \in \mathrm{c}[\mathrm{a}, \infty)$ and $\operatorname{Sup}\{|\mathrm{f}(\mathrm{x})|: \mathrm{x} \in[\mathrm{a}, \infty)\}=\mathrm{M}<\infty$. Choose $\lambda$ such that $\lambda<\left(e^{\alpha}\left(\frac{c}{\alpha}\right)^{\alpha}\right)^{-1}$. Then there exists a continuous vector function $\mathrm{y}(\mathrm{x})=\left(\mathrm{y}_{1}(\mathrm{x}), \mathrm{y}_{2}(\mathrm{x}), \ldots, \mathrm{y}_{\mathrm{n}}(\mathrm{x})\right)^{\mathrm{T}}$, $\mathrm{x} \in(\mathrm{a}, \infty)$ such that $y^{(\alpha)}(x)=\lambda f(y(x)), x \in(a, \infty)$ with $y^{(\alpha-1)}(a)=\mu$, where $\mu=\left(\mu_{1}, \mu_{2}, \ldots, \mu_{n}\right)^{T}$ is some constant vector and satisfies $|\mathrm{y}(\mathrm{x})|<\exp \left(\alpha \mathrm{c}^{-1}|\mathrm{x}|\right) \cdot$ constant.

\section{Proof}

Let $(\mathrm{A},\|\cdot\|)$ be the space defined in definition $(2.1),[\mathrm{a}, \mathrm{a}+\mathrm{h}]$ be compact subinterval of $[\mathrm{a}, \infty)$. Consider

$$
y(x)=y_{0}(x)+\frac{1}{\Gamma(\alpha)} \int_{a}^{x}(x-t)^{\alpha-1} f(y(t)) d t, x \in(a, \infty)
$$

where $y_{0}(x)=\left(\frac{\mu_{1}(x-a)^{a-1}}{\Gamma(\alpha)}, \cdots, \frac{\mu_{n}(x-a)^{a-1}}{\Gamma(\alpha)}\right)^{T}$, if follows from Lemma (2.1) that $\mathrm{y} \in(\mathrm{a}, \infty)$.

Then $(x-a)^{1-\alpha} y(x)=b+\frac{(x-a)^{1-\alpha}}{\Gamma(\alpha)} \int_{a}^{x}(x-t)^{\alpha-1} f(y(t)) d t, x \in(a, \infty)$, where

$$
b=\left(\frac{\mu_{1}}{\Gamma(\alpha)}, \frac{\mu_{2}}{\Gamma(\alpha)}, \cdots, \frac{\mu_{n}}{\Gamma(\alpha)}\right)^{T}
$$

Let $\mathrm{F}(\mathrm{x})=(\mathrm{x}-\mathrm{a})^{1-\alpha} \mathrm{y}(\mathrm{x}), \mathrm{x} \in(\mathrm{a}, \infty) \ldots(3.3)$, where $\mathrm{y}$ given in (3.2) and define $\mathrm{F}(\mathrm{x}, \mathrm{y}(\mathrm{t}))=(\mathrm{x}-\mathrm{a})^{1-\alpha} \mathrm{f}(\mathrm{y}(\mathrm{t})), \mathrm{a} \leq \mathrm{t}<\mathrm{x}<\infty$ ...(3.4), then from Lemma (2.3) we have $\mathrm{F} \in \mathrm{c}[\mathrm{a}, \infty)$.

Now define an operator $\mathrm{k}$ on $\mathrm{A}$ as 


$$
(k F)(x)=\frac{1}{\Gamma(\alpha)} \int_{a}^{x}(x-t)^{\alpha-1} F(x, y(t)) d t, x \in[a, a+h]
$$

Then

$$
\begin{aligned}
|(k F)(x)| & =\left|\frac{1}{\Gamma(\alpha)} \int_{a}^{x}(x-t)^{\alpha-1} F(x, y(t)) d t\right|, x \in[a, a+h] \\
\leq & \frac{1}{\Gamma(\alpha)} \int_{a}^{x}(x-t)^{\alpha-1} e^{-\gamma|y(t)|} e^{\gamma|y(t)|}|F(x, y(t))| d t
\end{aligned}
$$

and so from definition (1.2), we have

$$
|(k F)(x)| \leq \frac{1}{\Gamma(\alpha)} \int_{a}^{x}(x-t)^{\alpha-1} e^{\gamma|y(t)|}\|F\| d t
$$

Thus by using (3.1) we have

$$
\begin{aligned}
|(k F)(x)| & \leq \frac{\|F\|}{\Gamma(\alpha)} \int_{a}^{x}(x-t)^{\alpha-1} e^{\gamma(|t|+c)} d t \\
& =\frac{\|F\| e^{\gamma c}}{\Gamma(\alpha)} \int_{a}^{x}(x-t)^{\alpha-1} e^{\gamma|t|} d t \quad \cdots(3.6)
\end{aligned}
$$

Then from [10] we have

$$
\frac{1}{\Gamma(\alpha)} \int_{a}^{x}(x-t)^{\alpha-1} e^{\gamma|t|} d t=\gamma^{-\alpha} e^{\gamma|x|}
$$

Then from (3.6) and (3.7) we have

$|(k F)(x)|=\|F\| e^{\gamma c} \gamma^{-\alpha} e^{\gamma|x|}$

Thus $\quad|(k F)(x)| \leq\|F\| e^{\gamma c} \gamma^{-\alpha} e^{\gamma|x|}$

Hence $e^{-\gamma|x|}|(k F)(x)| \leq\|F\| e^{\gamma c} \gamma^{-\alpha}$ and so by definition (2.1) we get

$\|(k F)(x)\| \leq\|F\| e^{\gamma c} \gamma^{-c}$ and hence

$\|k\| \leq \gamma^{-\alpha} e^{\gamma c}$

Now for $|\lambda|<\mid\|\mathrm{k}\|^{-1}$ we obtain $\|\lambda \mathrm{k}\|=|\lambda|\|\mathrm{k}\|<|| \mathrm{k}\left\|^{-1}\right\| \mathrm{k} \|=1$ and this implies that on using Lemma (2.2) $\{\mathrm{I}-\lambda \mathrm{k}\}^{-1}$ exists. Also from (3.3) we have

$$
F(x)=b+\frac{\lambda}{\Gamma(\alpha)} \int_{a}^{x}(x-t)^{\alpha-1} F(x, y(t)) d t
$$

where $b=\frac{\mu}{\Gamma(\alpha)}$

Now (3.5) and (3.10) imply that

$\mathrm{F}(\mathrm{x})=\mathrm{b}+\lambda \mathrm{kF}(\mathrm{x})$, and so

$\mathrm{F}\{\mathrm{I}-\lambda \mathrm{k}\}=\mathrm{b}$ and therefore

$F(x)=\{I-\lambda k\}^{-1}(b)$ where $I$ is the identity operator and hence $F(x)$ exists and is the unique solution of

$$
F(x)=b+\frac{\lambda}{\Gamma(\alpha)} \int_{a}^{x}(x-t)^{\alpha-1} F(x, y(t)) d t
$$

Now by simple calculation one can show that 
$\min _{\gamma} \gamma^{-\alpha} e^{\gamma c}=e^{\alpha}\left(\frac{c}{\alpha}\right)^{\alpha}$ where $\gamma \mathrm{c}=\alpha$

Since from (3.9), $\|k\|<\frac{e^{\gamma c}}{\gamma c}$, there for

$\|k\|=\min _{\gamma}\left(\frac{e^{\gamma c}}{\gamma c}\right)=e^{\alpha}\left(\frac{c}{\alpha}\right)^{\alpha}$

Thus for

$|\lambda|<\frac{1}{e^{\alpha}\left(\frac{c}{\alpha}\right)^{\alpha}}$ the solution F of (3.10) exists and satisfies

$\mathrm{F}(\mathrm{x})=\mathrm{b}+\lambda \mathrm{kF}(\mathrm{x})$

Again from (3.3) we have

$F(x)=b+\frac{\lambda}{\Gamma(\alpha)} \int_{a}^{x}(x-t)^{\alpha-1} F(x, y(t)) d t$ for all $\mathrm{x} \in(\mathrm{a}, \mathrm{a}+\mathrm{h}]$

and by using (3.3), it follows that

$$
\begin{aligned}
& (x-a)^{1-\alpha} y(x)=\frac{\mu}{\Gamma(\alpha)}+\frac{\lambda(x-a)^{1-\alpha}}{\Gamma(\alpha)} \int_{a}^{x}(x-t)^{\alpha-1} F(y(t)) d t \text { for all } \mathrm{x} \in(\mathrm{a}, \mathrm{a}+\mathrm{h}] \\
& y(x)=\frac{\mu(x-a)^{\alpha-1}}{\Gamma(\alpha)}+\frac{\lambda}{\Gamma(\alpha)} \int_{a}^{x}(x-t)^{\alpha-1} F(y(t)) d t
\end{aligned}
$$

Therefore by definition (2.2) (a) we get

$$
y(x)=\frac{\mu(x-a)^{\alpha-1}}{\Gamma(\alpha)}+\lambda \prod_{a}^{\alpha} f \quad \mathrm{x} \in(\mathrm{a}, \mathrm{a}+\mathrm{h}]
$$

But from Lemma (2.4) we have $I_{a}^{x} \frac{(t-a)^{\alpha-1}}{\Gamma(\alpha)}=0$ and by Lemma (2.5) we get

$I_{a}^{x}{ }_{a}^{-\alpha} I^{\alpha} f=f(y(x))$ for all $\mathrm{x} \in(\mathrm{a}, \mathrm{a}+\mathrm{h}]$

Thus ${\underset{a}{a}}^{\alpha} y=\lambda f(y(x)) \mathrm{x} \in(\mathrm{a}, \mathrm{a}+\mathrm{h}]$

Then using definition (2.2)(b) we get

$$
y^{(\alpha)}(x)=I_{a}^{-\alpha} y=\lambda f(y(x))_{\mathrm{x} \in(\mathrm{a}, \mathrm{a}+\mathrm{h}]}
$$

Further more from (3.12) we have

$$
I_{a}^{x}{ }^{1-\alpha} y=I_{a}^{x} \frac{\mu(x-a)^{\alpha-1}}{\Gamma(\alpha)}+\lambda I_{a}^{x}{ }^{1-\alpha} I_{a}^{x} f
$$

It follows from Lemma (2.4) that 


$$
\begin{aligned}
\boldsymbol{I}_{a}^{x}{ }^{1-\alpha} y & =\mu+\lambda \prod_{a}^{x}{ }^{1-\alpha} I_{a}^{x}{ }^{\alpha} f=\mu+\lambda \prod_{a}^{x}{ }^{1} f \\
& =\mu+\lambda \int_{a}^{x} f(y(t)) d t
\end{aligned}
$$

and so $I_{a}^{x}{ }^{1-\alpha} y$ exists for all $\mathrm{x} \in(\mathrm{a}, \mathrm{a}+\mathrm{h}]$

since by definition (2.2)(b)

$$
\begin{aligned}
& y^{(\alpha-1)}(x)=I_{a}^{x}{ }^{(1-\alpha)} y, \text { therefore } \\
& y^{(\alpha-1)}(a)=\mu
\end{aligned}
$$

Now from (3.11) we have $|\mathrm{F}(\mathrm{x})| \leq \mathrm{b}+|\lambda||\mathrm{kF}(\mathrm{x})|$ and from (3.8) get

$$
\begin{aligned}
& |\mathrm{F}(\mathrm{x})| \leq \mathrm{b}+|\lambda|\|F\| \frac{e^{\gamma c}}{\gamma^{c}} e^{\gamma|x|} \\
& <\mathrm{b}+\|\boldsymbol{F}\| e^{\gamma|x|} \quad(\text { since } \gamma \mathbf{c}=\alpha) \\
& =e^{\gamma|x|}\left(\mathrm{b} e^{-\gamma|x|}+\|F\|\right) \\
& <e^{\gamma|x|}(\mathrm{b}+\|F\|)
\end{aligned}
$$

Thus by using (3.3) we obtain

$$
\begin{aligned}
& \left|(x-a)^{1-\alpha} y(x)\right|<e^{\gamma|x|}[b+\|F\|] \\
& h^{1-\alpha}|y(x)|<e^{\gamma|x|}[b+\|F\|] \\
& |y(x)|<e^{\gamma|x|} h^{\alpha-1}[b+\|F\|]
\end{aligned}
$$

and so the solution function satisfies $|y(x)|<\exp \left(x c^{-1}|x|\right) \cdot$ constant

\section{Conclusion}

In this study we prove the existence and uniqueness of solution for system of fractional differential equations using (theorem (1)) and (theorem (2)) in [9]. The solution obtained can used to solve many problems in the mathematic and other sciences such as mechanics engineering, chemistry, physics, etc.

\section{References}

[1] Sabatier, J. ;Agrawal, O.P.; Tenveiro Machado, J. A; Advances in Fractional Calculus: The oretical Developments and application in physics and Engineering, springer, 2007.

[2] Heymans, N.; Podlubny, I.; Physical Interpretation of Initial Conditions of Fractional Differential Equations with ReimannLiouville Fractional Derivatives. RheoLAcla, 45, 2006.

[3] Juan Bisquert, Albert compt. Theory of Electrochemical Impedance of an Amatous Diffusion, J of Electroanalytical chemistry 499, 2001.

[4] Barcenaetal, Discrete Control for Computer Hard Disc by Fractional Order Hold Devise. University of paisvais spain, 2000

[5] Shantana Das, B. B. Biswas, Total Energy Vtitization From Nuclear Sources PORT. 2006 Nuclear Energy for New Europe Slovenia, 2006.

[6] Shantana Das, etal. Ratio Control with Logarithmic Logies in new P and P control algorithm for a true fuel-efficient reactor. Int. J. Nuclear Energy science and Technology. Vol.3, No.1, 2007.

[7] V. Lakshmikantham, S. Leela and J. vasundhara; Theory of Fractional Dynamic Systems, Cambridge Academic publishes, Cambridge, 2009.

[8] Al-Tabakchaly, M. A., on the existence and uniqueness for fractional differential Equations, Msc. Thesis, University of Musol.

[9] Dr. Ahmed Zain-Abdin, Maha Ahd-Wahab, Theorem on Certain Fractional Functions and Derivative, Eng. and Tech. Journal. Vol.30, No.2, 2012.

[10] Spanier Keith B. Oldham and Jerome, The Fractional Calculus, Theory and Application of Differentiation to Arbitrary Order Manufactured in the United State by Courier Corporation: ISBN-13: 978-0-486-45001-8, 2006. 